\title{
Creating partially deleted objects for perception research
}

\author{
CHRISTOPHER KOCH, BRENT STRANDY, and BRENT WILSON \\ George Fox University, Newburg, Oregon
}

\begin{abstract}
Line drawings are commonly used in perception research. A basic strategy used in such research is to remove portions of the line drawings in order to determine what features of an object are important for recognition. However, it is important to monitor the amount of contour and type of information that are deleted when one is making partially deleted or fragmented objects. With the Image Fragmenting Program, researchers can use random or manual contour deletion strategies to create fragmented objects while controlling for the amount of contour removed from the images.
\end{abstract}

Line drawings of objects are frequently used in perception research. Such line drawings are often partially deleted or fragmented in order to study what features are important for object recognition. Biederman (1987), for example, removed full geons or either the vertices or midsegments of geons from simple line drawings to find evidence for recognition-by-components theory. Other researchers have created degraded line drawings by randomly deleting contour from the line drawings in order to study perception and memory (e.g., Snodgrass \& Hirshman, 1994). In fact, Snodgrass, Smith, Feenan, and Corwin (1987) describe a computer algorithm that randomly deletes pixels from line drawings. Snodgrass has also normed line drawings fragmented with this method (Snodgrass \& Vanderwart, 1980).

Regardless of how the line drawings are fragmented, an important factor to control is the amount of contour deleted from the object. Contour, especially contour providing information regarding the borders or outlines of objects, is important for recognition (see Lloyd-Jones \& Luckhurst, 2002). The amount of contour removed also affects object recognition across ages (see, e.g., Murray \& Szymczyk, 1978). Controlling the amount of contour deleted from a line drawing can be a tedious task. Contour can be measured manually using a stadiometer. However, contour can also be measured digitally. In a digital black and white line drawing, the number of black pixels can be counted, and as contour is deleted, the number of black pixels will decrease and the percent of contour deletion can be calculated.

\section{Image Fragmenting Program}

The Image Fragmenting Program (IFP) was developed to create fragmented digital line drawings for use in perception research. The line drawings can be modified with

Correspondence concerning this article should be addressed to C. Koch, Department of Psychology, 414 N. Meridian St., No. 6143, Newburg, OR 97132 (e-mail: ckoch@georgefox.edu). an automatic random deletion process or by hand. This is done with an eraser tool, which provides 10 eraser sizes. The smallest is $2 \times 2$ pixels; the erasers increase in size by even increments (i.e., $4 \times 4,6 \times 6,8 \times 8, \ldots, 20 \times 20$ ) and are able to erase a total of $16-400$ pixels. With the random deletion process, one can preset the amount of contour deletion to make numerous versions of a line drawing that vary in the amount of fragmentation. The user simply selects the eraser size and percent deletion by manipulating two slide bars (Figure 1). To create an image with $50 \%$ deletion, for instance, the user first chooses the eraser size (e.g., $4 \times 4)$ and sets the deletion slide bar to $50 \%$. The deletion process then randomly removes $4 \times 4$ pixel blocks until $50 \%$ of the contour has been deleted. Pixel squares are randomly selected with replacement, so it is possible that the eraser may either totally or partially overlap a previously deleted portion of a line drawing on any given "trial." However, the total amount of contour deleted is the focus of this process.

To purposely fragment a line drawing in order to remove certain information while maintaining other information, the researcher can manually delete contour. A pixel counter, indicating the percent of contour deleted, provides a running total of the number of pixels removed from a line drawing and the corresponding percent of the image deleted. With this method, the user can once again select the eraser tool size and remove contour by left clicking the mouse over the portion of contour to be removed. The counter changes with each pixel removed from a line drawing, and the corresponding percentage of fragmentation is presented (Figure 2).

Newly created images can be saved in relation to the original image somewhat as text can be saved as a thread to see how a conversation has progressed. Thus, a complete line drawing can serve as the original image, and IFP can be used to create different images, varying in the amount of contour deletion, that are linked to the parent image. Images can also be placed into category types. For example, a desk and chair can be placed in a furniture category and a duck and beaver can be placed in an ani- 


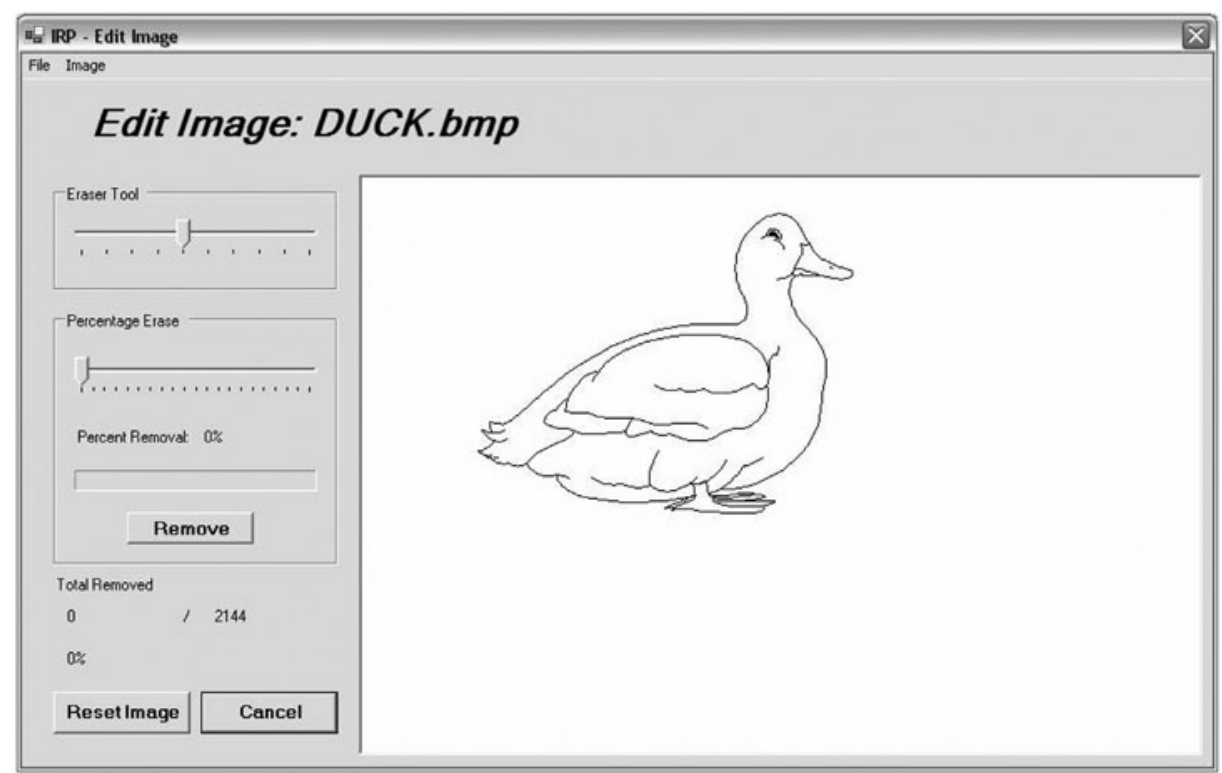

Figure 1. Editing screen in IFP showing the eraser (erase tool) and percent deletion (percent erase) slide bars.

mal category. Each time an image is saved, the filename, parent file, category type, and percent contour removed are recorded. This information appears in a file properties window when one views the list of file names from the main IFP window.

\section{.NET Framework}

IFP was created within the .NET framework. The .NET platform is a development framework that allows the programmer to utilize the application programming interfaces
(APIs) of Windows and incorporate distinct technologies such as $\mathrm{COM}+, \mathrm{XML}$, object-oriented design, and Web service protocols such as SOAP, WSDL, and UDDI. This framework sits on top of the operating system, which can be any version of Windows and potentially any version of Unix or other operating systems due to the common language runtime architecture. C\#, Visual Basic .NET, Managed $\mathrm{C}++$, and Jscript .NET are supported within the .NET framework. This support is based on common language specification (CLS), which allows flexibility in

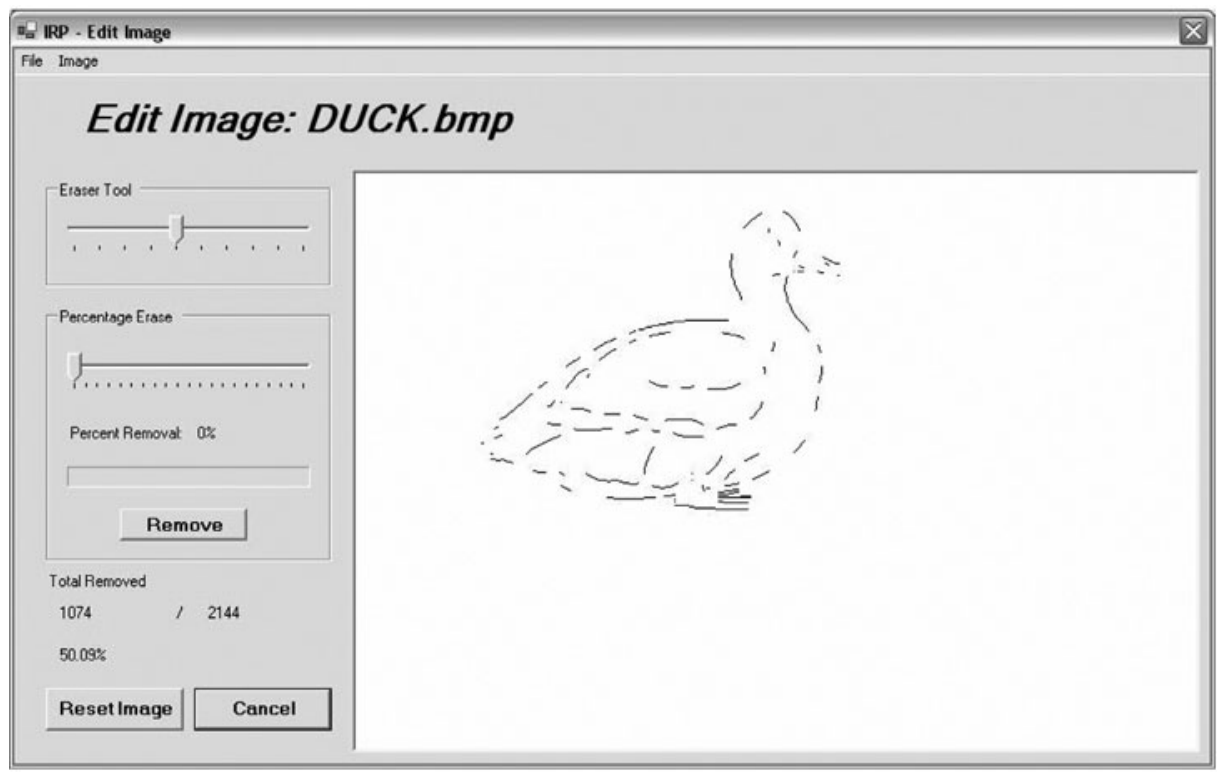

Figure 2. Edit screen in IFP after a line drawing of a duck was deleted by hand with $50 \%$ deletion. The percent deletion is presented in the bottom left corner of the screen. 
development since applications can be combined from several languages. In addition, framework base classes support input and output, string manipulation, security management, network communication, thread management, text manipulation, reflection, and collections functionality. Data and XML classes are also supported within the framework. Finally, .NET incorporates window forms, Web forms, and Web services technologies. Thus, the multitier framework of .NET allows developers to create applications from several languages, for multiple platforms, and with Web functionality. Additional information about .NET can be found at the Microsoft Developers Center (msdn.microsoft.com).

IFP was created with Visual Basic .NET in order to take advantage of the .NET framework. Specifically important for IFP are the thread management and database support of .NET, which provide a flexible structure for maintaining a catalog of images. Although IFP is currently a stand-alone program for desktop use, the Web support within .NET makes it possible to incorporate IFP with Web-based databases (e.g., Online Picture Database for Visual Perception Research; Koch \& Hansen, 2006). Finally, .NET allows IFP to be used with any version of Windows as well as with other operating systems.

\section{Summary}

IFP is a useful program for perception researchers who need to create partially deleted line drawings. The pro- gram is compatible with multiple operating systems, and it provides some database functionality for managing images. IFP is available online at cognition.georgefox.edu.

\section{REFERENCES}

BiEdERMAN, I. (1987). Recognition-by-components: A theory of human image understanding. Psychological Review, 94, 115-217.

Koch, C., \& Hansen, D. (2006). Online picture database for perception research. Manuscript submitted for publication.

LlOYD-JONES, T. J., \& LUCKHURST, L. (2002). Outline shape is a mediator of object recognition that is particularly important for living things. Memory \& Cognition, 30, 489-498.

MurRay, F. S., \& SZYMcZYK, J. M. (1978). Effects of distinctive features on recognition of incomplete pictures. Developmental Psychology, 14, 356-362.

SnODGRass, J. G., \& Hirshman, E. (1994). Dissociations among implicit and explicit memory tasks: The role of stimulus similarity. Journal of Experimental Psychology: Learning, Memory, \& Cognition, 20, $150-160$.

Snodgrass, J. G., Smith, B., Feenan, K., \& Corwin, J. (1987). Fragmenting pictures on the Apple Macintosh computer for experimental and clinical applications. Behavior Research Methods, Instruments, \& Computers, 19, 270-274.

SNOdgrass, J. G., \& VANDERWART, M. (1980). A standardized set of 260 pictures: Norms for name agreement, image agreement, familiarity, and visual complexity. Journal of Experimental Psychology: Human Learning \& Memory, 6, 174-215.

(Manuscript received November 16, 2005; revision accepted for publication April 26, 2006.) 